\title{
Balancing Cryptoassets and Gold: A Weighted-Risk-Contribution Index for the Alternative Asset Space
}

\author{
Aikaterini Koutsouri ${ }^{1}$, Francesco Poli $^{2}$, Elise Alfieri ${ }^{3}$, Michael Petch ${ }^{4}$, Walter \\ Distaso $^{5}$, and William J. Knottenbelt ${ }^{6}$ \\ 1 Department of Computing, Imperial College London, London, SW7 2AZ, UK \\ k.koutsouri@imperial.ac.uk \\ 2 Department of Finance, Imperial College Business School, London, SW7 2AZ, UK \\ f.poli@imperial.ac.uk \\ 3 Univ. Grenoble Alpes, 38000 Grenoble, France \\ Elise.Alfieri@univ-grenoble-alpes.fr \\ 4 CoinShares, Octagon Point, 5 Cheapside, St Paul's, London, England, EC2V 6AA \\ mpetch@coinshares.co.uk \\ 5 Department of Finance, Imperial College Business School, London, SW7 2AZ, UK \\ w.distaso@imperial.ac.uk \\ ${ }^{6}$ Department of Computing, Imperial College London, London, SW7 2AZ, UK \\ wjk@doc.ic.ac.uk
}

\begin{abstract}
Bitcoin is foremost amongst the emerging asset class known as cryptoassets. Two noteworthy characteristics of the returns of nonstablecoin cryptoassets are their high volatility, which brings with it a high level of risk, and their high intraclass correlation, which limits the benefits that can be had by diversifying across multiple cryptoassets. Yet cryptoassets exhibit no correlation with gold, a highly-liquid yet scarce asset which has proved to function as a safe haven during crises affecting traditional financial systems. As exemplified by Shannon's Demon, a lack of correlation between assets opens the door to principled risk control through so-called volatility harvesting involving periodic rebalancing. In this paper we propose an index which combines a basket of five cryptoassets with an investment in gold in a way that aims to improve the risk profile of the resulting portfolio while preserving its independence from mainstream financial asset classes such as stocks, bonds and fiat currencies. We generalise the theory of Equal Risk Contribution to allow for weighting according to a desired level of contribution to volatility. We find a crypto-gold weighting based on Weighted Risk Contribution to be historically more effective in terms of Sharpe Ratio than several alternative asset allocation strategies including Shannon's Demon. Within the crypto-basket, whose constituents are selected and rebalanced monthly, we find an Equal Weighting scheme to be more effective in terms of the same metric than a market capitalisation weighting.
\end{abstract}

Keywords: Cryptoassets · Index · Volatility · Rebalancing Premium . Risk Management · Asset Allocation · Equal Risk Contribution · Weighted Risk Contribution · Bitcoin · Gold 


\section{Introduction}

Cryptoassets are increasingly recognised as viable investments. Since they exhibit little correlation with traditional asset classes [1], adding even a small percentage of cryptoassets to a portfolio can enhance risk-adjusted returns [6] while also offering a safe haven in the event of a financial crisis. Until recently, the cryptoasset market was afflicted by a lack of clear regulatory guidance, with uncertainty surrounding the classification of the asset, its tax treatment, and the effect of events specific to the cryptocurrency domain such as forks. However, the last year has seen the emergence of more mature and well-defined regulatory frameworks $[11,17,20]$. In turn, this has driven increased institutional demand for cryptoasset-based financial indicators.

Regardless of the creation of new financial products, many investors still see the crypto market as being unacceptably risky due to its high volatility - something not unusual for an emerging asset class. Although volatility poses challenges in terms of increased uncertainty, there are also benefits to be had from its proper management through diversification and regular rebalancing [4]. This is exemplified by the so-called Shannon's Demon approach in which two, ideally uncorrelated, assets - at least one of which is highly volatile - are periodically rebalanced to maintain an ideal target allocation. The resulting expected growth rate is greater than the arithmetic mean of the individual expected growth rates, while the variance of the returns is less than the mean of the individual variances [15, pp. 201-209].

In theory, this strategy would be well-suited for the volatile cryptoasset class and an uncorrelated wealth-preserving asset class. Although there are plenty of candidates uncorrelated with cryptoassets, not all are properly suited. For example, traditional wealth-preserving assets such as property or museum-quality fine art are illiquid [16]. An asset such as gold is much more appropriate because of its low volatility, high liquidity and ability to act as a hedge to traditional financial markets $[10,1,5]$. Gold is also more suitable in this context than other precious metals such as platinum or silver, since the latter, unlike gold, have not historically served as a hedge or safe haven during times of financial turmoil [12].

Pure-crypto indices such as CRIX [21], CRYPTO20 [7], MVDA5 [14], and Bloomberg Galaxy Crypto Index [2] do offer broad exposure to the cryptomarket. However, they are characterised by a volatility close to that of the single cryptoassets. Thus, they do not incorporate mechanisms for effective risk control beyond simple diversification over their (highly-correlated) constituents.

By contrast, the purpose of this study, put forth jointly by researchers at Imperial College London and CoinShares, is to propose a low-volatility index that combines an uncorrelated asset (gold) with a basket of cryptoassets, using weighted-risk contribution as a rebalancing mechanism. By decreasing volatility levels, it yields superior risk-adjusted returns when compared to a number of alternative strategies, including holding cryptoassets or gold alone. Further, the proposed index presents a moderate turnover, which translates into moderate operating costs. A fuller index methodology document, together with a reference implementation, will be made available online in due course. 
The remainder of this paper is organised as follows. Section 2 presents background related to portfolio diversification, while Section 3 extends the theory of Equal Risk Contribution to Weighted Risk Contribution, in which the contribution to volatility by two uncorrelated asset classes (cryptoassets and gold in our case) can be varied to taste. Section 4 presents an overview of the index methodology. Section 5 demonstrates the historical performance of the index, presenting an improved risk and returns profile compared to other established methodologies. Section 6 concludes.

\section{Background}

\subsection{Shannon's Demon}

In the 1960s, Claude Shannon presented an optimal growth-portfolio construction method exploiting diversification and rebalancing (cf. $[3,19])$. This method, called Shannon's Demon [15], considers two assets: a highly volatile one that follows a pure random walk process, which can either double in price or drop by $50 \%$, and an uncorrelated low-volatility one, specifically cash. In the proposed portfolio half of the capital is allocated to the volatile asset and half to cash.

The general setting is: an investor decides at the beginning which fraction of his initial wealth to put at risk and then regularly rebalances between the risky asset and cash taking into account the proceeds in the game. The accumulated wealth after $T$ rounds and $L$ losses is $W_{T}=W_{0}\left[\left(1+w_{0} a\right)^{(T-L)}\left(1-w_{0} b\right)^{L}\right]$, where $W_{0}$ is the initial wealth, $w_{0}$ the fraction reallocated in risk, $a$ the percentage returns in an up-move proportional gain and $b$ the percentage loss in a downmove proportional loss. The objective is to maximise the log utility of wealth, which implies maximising the expected growth rate:

$$
E[g]=p \log \left(1+w_{0} a\right)+q \log \left(1-w_{0} b\right)
$$

where $p$ is the probability of profit and $q$ is the probability of loss. The optimal fraction to invest in the risky asset is then:

$$
w_{0}^{*}=\frac{p a-q b}{a b}
$$

The conditions under which a rebalancing approach outperforms buy-andhold are explored in [9]. The same work considers a case of two negativelycorrelated volatile assets. It is shown that even with diversification and positive expected returns, a buy-and-hold strategy can fail to grow an investor's wealth. Active management by rebalancing, on the other hand, builds long-term value.

Shannon's scheme is a strategy that can generate growth even if the returns of both assets are negative. It provides a solution to Parrondo's Paradox [18] which states that a winning strategy can emerge from the intelligent combination of two losing strategies. More generally, it provides a means to harness high volatility and low correlation in a way that reduces portfolio risk through diversification and rebalancing [4]. However, there is a trade-off between the frequency of rebalancing (which should be high to lead to a higher growth rate) and turnover (which should be kept moderate to avoid high transaction costs). 


\subsection{Equal Risk Contribution}

Risk-based strategies have proved to be capable of reducing volatility in a way that does not impede market exposure, while outperforming standard strategies in unsteady markets. Equal Risk Contribution (also known as Risk Parity) is a well known risk-control strategy that achieves diversification both within and across asset classes. Its main goal is to bolster the portfolio's immunity to unforeseen drawdowns during stressful market periods. In contrast with the equally-weighted allocation scheme, a Risk Parity portfolio aims towards an equal distribution of the overall budget, expressed in terms of risk rather than capital. As a result, it achieves better risk-adjusted returns.

The Risk Parity optimisation problem setting is constituted of $N \geq 2$ assets $A_{1}, \ldots, A_{N}$, with $\mu_{i}, \sigma_{i}$ and $\sigma_{i}^{2}$ representing the expected return, standard deviation and variance of the returns of $A_{i}$ respectively and $\rho_{i j}$ denoting the correlation coefficient of the returns of $A_{i}$ and $A_{j}$ for $i \neq j$. The $N \times N$ symmetric covariance matrix of returns is defined as $\Sigma=\left(\sigma_{i j}\right)$ where $\sigma_{i j}=\rho_{i j} \sigma_{i} \sigma_{j}, i \neq j$ and $\sigma_{i j}=\sigma_{i}^{2}, i=j$. If $x_{i}$ is the amount to be invested in asset $A_{i}$, then the volatility (measured in terms of standard deviation) of the resulting portfolio $x=\left(x_{1}, \ldots, x_{i}\right)$ is computed as $\sqrt{x^{T} \Sigma x}$.

In the Equal Risk Contribution problem, $\sigma(x)=\sqrt{x^{T} \Sigma x}$ denotes the risk of the portfolio, and through the Euler decomposition, the risk is expressed as:

$$
\sigma(x)=\sum_{i=1}^{N} \sigma_{i}(x)=\sum_{i=1}^{N} x_{i} \times \frac{\partial \sigma(x)}{\partial x_{i}},
$$

where $\partial_{x_{i}} \sigma(x)$ is the marginal risk contribution and $\sigma_{i}(x)=x_{i} \times \partial_{x_{i}} \sigma(x)$ is the (total) risk contribution of asset $A_{i}$. The desired risk-balanced portfolio is constituted in a way that all components contribute equally to the overall volatility; therefore $\sigma_{i}(x)=\sigma_{j}(x)$. The general Risk Parity portfolio construction problem can be mathematically expressed as:

$$
x_{E R C}=\left\{x \in[0,1]^{N}: x_{i} \times \partial_{x_{i}} \sigma(x)=x_{j} \times \partial_{x_{j}} \sigma(x), \forall i, j, \sum_{i=1}^{N} x_{i}=1\right\} .
$$

Through the problem expression, asset classes with reduced levels of volatility or correlation are favoured since their marginal risk contribution to the portfolio volatility will be lower. In [13], Maillard et al. show that if all correlations are the same then each constituent weight is defined as the ratio of the reciprocal of its volatility with the sum of the reciprocals of the volatilities of all constituents:

$$
x_{i}=\frac{\sigma_{i}^{-1}}{\sum_{j=1}^{N} \sigma_{j}^{-1}}, i=1, \ldots, N
$$

and therefore, in the bivariate case,

$$
x_{1}=\frac{\sigma_{1}^{-1}}{\sigma_{1}^{-1}+\sigma_{2}^{-1}} .
$$


In [13], when the correlations are different, the authors propose solving the optimisation problem defined as

$$
\min _{x} \sum_{i=1}^{N} \sum_{j=1}^{N}\left(x_{i}(\Sigma x)_{i}-x_{j}(\Sigma x)_{j}\right)^{2}
$$

with $x_{i} \in[0,1]$ and $\sum_{i=1}^{N} x_{i}=1$. Here $(\Sigma x)_{i}$ denotes the $i^{\text {th }}$ entry of the vector resulting from the product of $\Sigma$ with $x$.

\section{Weighted Risk Contribution}

One potential concern about the classic Equal Risk Contribution scheme is that, because it belongs to the family of inverse volatility weighting, it can potentially generate allocations that are too concentrated towards assets with low volatility or low correlation, causing the undesired effect of lowering the degree of diversification inside a portfolio when no constraints are introduced.

This is indeed what happened to many Risk Parity funds in the last two years. In fact, given the low rates set by Central Banks in the most advanced economies, sovereign bonds returns reached an unprecedented low level of volatility and an unconstrained minimisation resulted in an extremely high weight for this asset class. When Central Banks moved on to raise rates, Risk Parity portfolios found themselves too exposed to that risk and suffered important losses. In this case, when it comes to the weighting of cryptoassets alone, the risk of a similar scenario is somehow less of a concern, because of the similar level of volatility between cryptoassets and because of their high level of correlation.

We address this issue by allowing the proportion of risk contribution by each asset class to be configurable. Following [13], the vector of risk contributions in the two-asset case given weighting $x=\left(x_{1}, x_{2}\right)$ and correlation $\rho$ is:

$$
\frac{1}{\sigma(x)}\left(\begin{array}{c}
x_{1}^{2} \sigma_{1}^{2}+x_{1} x_{2} \rho \sigma_{1} \sigma_{2} \\
x_{2}^{2} \sigma_{2}^{2}+x_{1} x_{2} \rho \sigma_{1} \sigma_{2}
\end{array}\right)
$$

Considering the case of uncorrelated assets $(\rho=0)$, and supposing that we desire the risk contribution of asset 1 to be $\alpha$ times the risk contribution of asset 2 , we need to solve for $x_{1}$ in:

$$
x_{1}^{2} \sigma_{1}^{2}=\alpha\left(x_{2}^{2} \sigma_{2}^{2}\right)
$$

Given $x_{i} \in[0,1]$ and $\sum_{i=1}^{2} x_{i}=1$ this yields:

$$
x_{1}=\frac{\sqrt{\alpha} \sigma_{1}^{-1}}{\sqrt{\alpha} \sigma_{1}^{-1}+\sigma_{2}^{-1}}
$$

In our case, $x_{1}$ represents the proportion of the investment allocated towards a basket of cryptoassets whose components are equally weighted, while $x_{2}$ is the proportion invested in gold. The risk contribution ratio is set as $\alpha=4$, indicating that $80 \%$ of the total risk emanates from the crypto-basket. 


\section{Index Overview}

\subsection{Design Goals}

The objective of this study is the design and implementation of an index that should meet the following goals:

1. Provide exposure to the alternative asset space in a way that is orthogonal to traditional financial markets;

2. Be comprised of a small number of liquid, investable constituent assets;

3. Exhibit a relatively stable composition in terms of constituents with asset weights that do not vary dramatically between rebalancing periods, leading to low or moderate turnover;

4. Utilise some means of principled risk control leading to lower volatility;

5. Be specified in a clear and unambiguous manner to facilitate validation and reproducibility;

6. Hold constituent assets on a long-only basis;

7. Not make use of leverage.

In terms of Goal 4, historical volatility of cryptoassets has remained at much higher levels compared to other asset classes while correlation among single non-stablecoin cryptoassets is persistent, displaying some signs of time variability. Therefore, constructing an index constituted only of cryptoassets offers very little prospect of diversification irrespective of the methodology used and hence, less prospect of bringing down its volatility. Gold returns, on the other hand, have been much less volatile than those of cryptoassets and have displayed a very low time varying correlation with cryptoassets (see Fig. 1). Gold was therefore the ideal candidate to include alongside cryptoassets with the purpose of considerably reducing volatility.

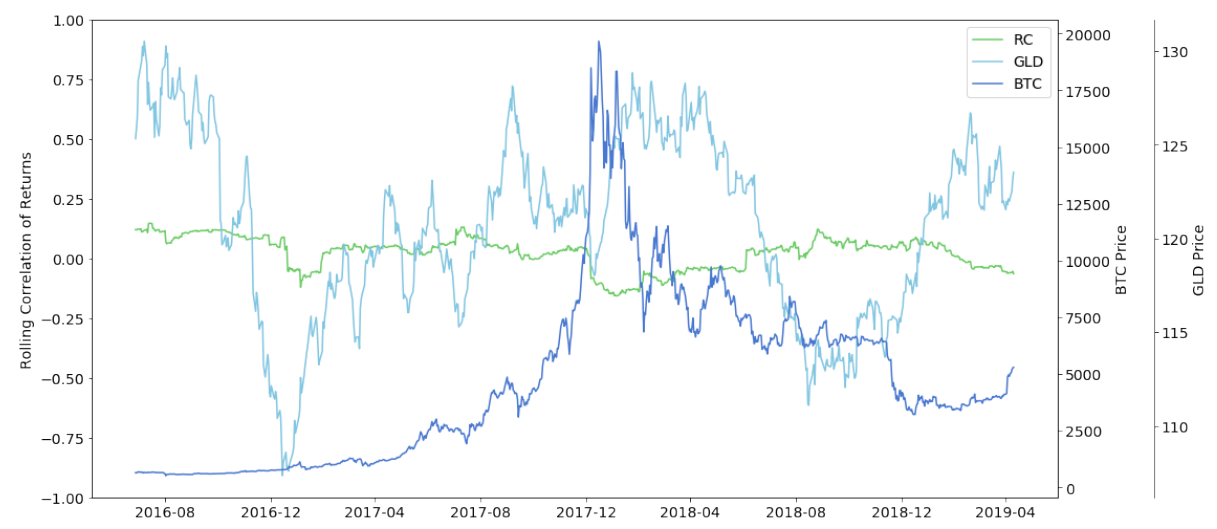

Fig. 1. 180-Day Rolling correlation (RC) between daily returns of Bitcoin (BTC) and Gold (GLD) 


\subsection{Constituent Eligibility and Selection}

The index is composed of a fixed number of constituents including five cryptoassets and SPDR Gold Shares (GLD), the largest gold ETF. The cryptoasset constituents of the index are the top five eligible cryptoassets based on the 6month rolling mean of free-float market capitalisation. By restricting the index to the top five cryptoassets we are less likely to encounter liquidity issues. Selection of constituents occurs on a monthly basis.

We determine whether a cryptoasset is eligible to be selected, based on the following requirements:

1. Trades in USD;

2. Is not linked to the value of a fiat currency;

3 . Has at least a 6 -month history of trading on a reputable exchange;

4. Has been on its native blockchain for at least 6 months;

5. Is not an ERC20 token;

6. Is not a privacy-focused coin (e.g. Monero, ZCash);

7. Has not suffered a major chain reorganisation in the last 6 months, and is not subject to a forthcoming contentious hard fork before the next selection is due to take place.

\subsection{Constituent Weighting}

For the weighting of the constituents, we choose a bi-level approach that involves studying the historical volatilities of the crypto-basket and gold separately in order to inform the crypto-gold asset allocation decision. That is because if GLD is added to a basket of five cryptoassets for a global allocation scheme, the correlation structure between all six assets cannot be ignored and the constituents' weighting procedure cannot be performed through Eq. (1). Also, in order to be able to produce a robust estimation of covariance matrices, the behaviour of the two asset classes would have to be studied only in time spans where exchanges for both are open. The bi-level approach on the other hand allows for exploitation of all available market data.

Regarding the formation of the crypto-basket, due to the persistent levels of correlation between non-stablecoin cryptoassets, any Risk Parity approach is expected to lean towards an Equally Weighted allocation whose risk level is not significantly improved. Therefore, due to its much more convenient reproducibility compared to Eq. (2) and the fragility of Eq. (2) when the covariance matrix is barely positive semi-definite, an Equally Weighted scheme is employed within the crypto-basket.

Taking into consideration the former, and the lack of a significant correlation between gold and cryptoassets, the index is calculated following a two-stage allocation scheme that involves:

1. Computation of the historical volatility of (a) the equally weighted cryptobasket, and (b) gold;

2. Asset allocation among the crypto-basket and gold expressed as the bivariate weighted risk contribution problem presented in Section 3. 


\subsection{Rebalancing Schedule}

In order to capture the diversification benefits of the time-varying correlations between gold and crypto highlighted in Figure 1, we have chosen a monthly rebalancing frequency. Coupled with the monthly reselection it allows the index to represent the rapidly evolving market conditions. This has no dramatic impact on the turnover of the portfolio and hence keeps transaction costs to an acceptable level.

\subsection{Index Calculation}

The Index base level is set on 1000 on January 1st, 2016:

$$
\text { Index }_{0}=1000
$$

The Index level on day $t$ from January 2nd, 2016 onwards is calculated as:

$$
\operatorname{Index}_{t}=\frac{\sum_{i \in N_{t}} P_{i, t} \times x_{i, t}}{D_{t}}
$$

where

- $N_{t}$ is the set of the 6 selected assets ( 5 cryptocurrencies and gold) on day $t$

- $P_{i, t}$ is the closing price for asset $i$ on day $t$ expressed in USD

- $x_{i, t}$ is the weight of asset $i$ on day $t$ as computed through the WRC allocation scheme at the beginning of the month

- $D_{t}$ is the Index Divisor on day $t$

The Index Divisor is used so that assets weight rebalancing and substitution do not alter the Index level. It is calculated using the following formula:

$$
D_{t}=\frac{\sum_{i \in N_{t}} P_{i, t-1} \times x_{i, t}}{\sum_{i \in N_{t-1}} P_{i, t-1} \times x_{i, t-1}} \times D_{t-1}
$$

The Divisor on January 2nd, 2016 is calculated as:

$$
D_{1}=\frac{\sum_{i \in N_{1}} P_{i, 0} \times x_{i, 1}}{1000}
$$

where

- $N_{1}$ is the set of the selected assets on January 2nd, 2016

- $P_{i, 0}$ is the closing price for asset $i$ on January 1st, 2016 expressed in USD

- $x_{i, 1}$ is the weight of asset $i$ on January $2 \mathrm{nd}, 2016$

Equations (5-7) are equivalent to computing recursively the value of the Index using the weighted average of its constituent's returns:

$$
\text { Index }_{t}=\sum_{i \in N_{t}} \frac{P_{i, t}}{P_{i, t-1}} x_{i, t} \times \sum_{i \in N_{t-1}} \frac{P_{i, t-1}}{P_{i, t-2}} x_{i, t-1} \times \cdots \times \sum_{i \in N_{1}} \frac{P_{i, 1}}{P_{i, 0}} x_{i, 1} \times 1000
$$


which implies

$$
\text { Index }_{t}=\sum_{i \in N_{t}}\left(1+R_{i, t}\right) x_{i, t} \times \text { Index }_{t-1}, \quad t=1,2, \ldots
$$

where

- $R_{i, t}$ is the return of asset $i$ from time $t-1$ to time $t$

- Index 0 is the base level of the Index set at 1000 on January 1st, 2016

Figure 2 shows how the index value would have evolved over the period January 2016 to April 2019. A detailed breakdown and comparison of index performance is presented in Section 5 .

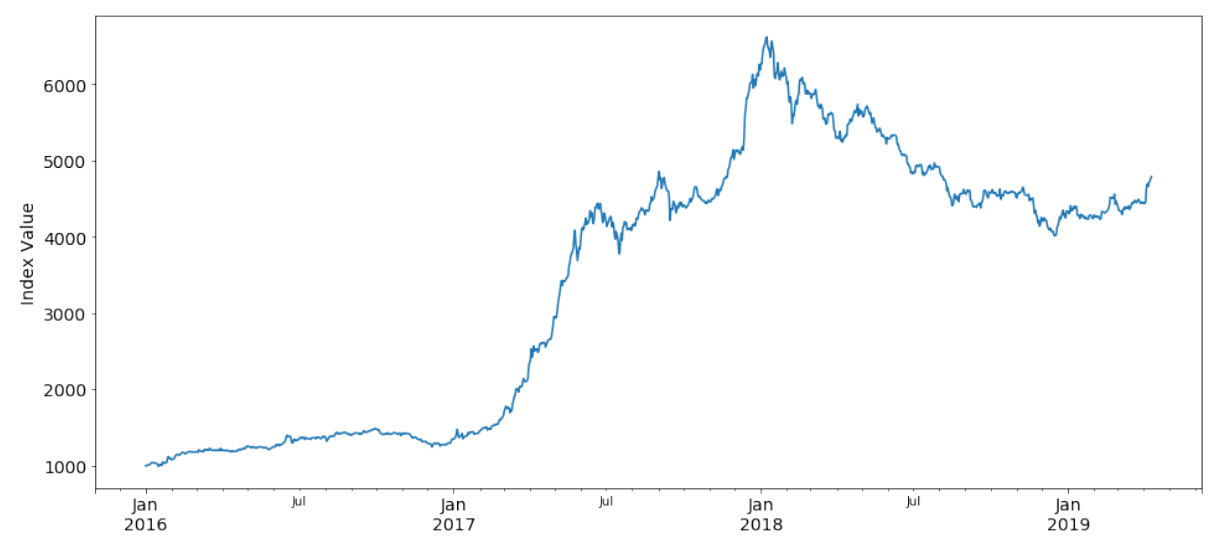

Fig. 2. Index Value January 2016-April 2019

\subsection{Hard Fork and Airdrop Policy}

Hard Fork Policy A 'Hard Fork' occurs when a change is made to the transaction validation rules of a cryptoasset's underlying blockchain protocol in a way that is not compatible with its earlier version. Nodes that wish to continue to participate are expected to upgrade to the new version of the protocol's software. Usually such a fork is planned and accepted by the overwhelming majority of nodes. However, where the fork is contentious enough that a non-negligible number of nodes continue to run the old version of the software, a chain split occurs.

The index will feature a Governing Committee which will evaluate all upcoming hard forks, especially in light of Rule 7 of Section 4.2. Treatment of hard forks will be led by decisions of exchanges with respect to the ticker symbols used to represent the resulting cryptoassets and the markets that they 
maintain. Concretely, suppose some cryptoasset traded under ticker symbol $T$ is expected to undergo (or undergoes) a hard fork resulting in an original chain $C$ with cryptoasset $C_{a}$ and a modified chain $C^{\prime}$ with cryptoasset $C_{a}^{\prime}$. There are a few scenarios to consider:

- $C_{a}$ continues to trade under ticker symbol $T$ while $C_{a}^{\prime}$ starts trading under a newly-created ticker symbol $T^{\prime}$. The BTC-BCH fork is an example of this scenario. In this case, $C_{a}$ continues as a constituent of the index. $C_{a}^{\prime}$ is not eligible to become a constituent of the index (lacking as it does the necessary pricing history), and does not contribute to the index value. $C_{a}^{\prime}$ may be sold by funds tracking the index as an excess return; the precise decision of when (or whether) to sell will be a matter of judgment for the tracking funds.

- $C_{a}^{\prime}$ now trades under ticker symbol $T$ while $C_{a}$ starts trading under a new ticker symbol $T^{\prime}$. The ETH-ETC fork is an example of this scenario. In this case, $C_{a}^{\prime}$ replaces $C_{a}$ as a constituent of the index. The pricing history for $C_{a}^{\prime}$ is taken as being that of $C_{a}$ prior to the fork. $C_{a}$ is no longer a constituent of the index, does not contribute to the index value, and may be sold by funds tracking the index as an excess return.

- $C_{a}^{\prime}$ now trades under ticker symbol $T$ while trading in $C_{a}$ is (largely) abandoned. Hard forks to upgrade the consensus mechanism of Monero usually follow this pattern. In this case, $C_{a}^{\prime}$ replaces $C_{a}$ as a constituent of the index and the pricing history for $C_{a}^{\prime}$ is taken as being that of $C_{a}$ prior to the fork.

- There is substantial disagreement amongst exchanges as to the ticker symbols that $C_{a}^{\prime}$ and $C_{a}$ should trade under. Usually this scenario would arise as the result of a contentious hard fork. Since cryptoassets due to undergo contentious hard forks before the next selection date are not eligible for selection, it is expected that this situation would apply to index constituents only in very rare circumstances. In this case, an extraordinary meeting of the Governing Committee will be convened in order to decide on an appropriate course of action which may include replacing $C_{a}$ by the next eligible cryptoasset, or rebalancing across the remaining constituent cryptoassets.

Airdrop Policy An Airdrop occurs when a blockchain project distributes free cryptoassets to investors in the hopes of attracting more people to use their platform. Occasionally some projects offer more established cryptoassets to do an Airdrop but most of the time, the project Airdrops their own native token or cryptocurrency. Requirements to qualify for an Airdrop vary as well; in some cases the participant has to hold the cryptoasset in their wallet while other times they have to promote the project on an online forum.

Airdropped cryptoassets will not be included in the index. Fund managers tracking the index may sell these at their earliest convenience, thus contributing to excess returns over the base index. 


\section{Results}

\subsection{Methodology and Data Source}

In order to evaluate the effectiveness of a Weighted Risk Contribution (WRC) strategy in the cryptoasset and gold case, the performance of a respective risk distribution portfolio is measured and compared against various strategies including buy-and-hold bitcoin (BTC), buy-and-hold gold (GLD), market capitalisation weighted pure cryptoassets, Shannon's Demon using bitcoin and gold, and Equal Risk Contribution (ERC) cryptoassets. The dataset used for the implementation and backtesting of the described allocation method includes daily values of historical free float market capitalisation and USD prices for more than 3000 cryptoassets, obtained from CoinGecko as well as daily adjusted USD prices of SPDR Gold Shares (GLD). The backtest that is performed covers the period between January 2016 and April 2019, a time span that reflects a wide variety of market conditions for the cryptoasset space. The datasets produce daily returns for both asset classes and assumes monthly rebalancing for all active strategies.

Table 1 shows the results of monthly selection of cryptoasset constituents that meet the eligibility criteria. Note that only dates where the constituents change are presented.

\begin{tabular}{||lccccc||}
\hline Date & Constituent 1 & Constituent 2 & Constituent 3 & Constituent 4 & Constituent 5 \\
\hline \hline $2016-01-01$ & Bitcoin (BTC) & Ripple (XRP) Litecoine (LTC) & Dash (DASH) & Dogecoin (DOGE) \\
\hline $2016-03-01$ & BTC & XRP & LTC & Ethereum (ETH) & DASH \\
\hline $2017-02-01$ & BTC & ETH & XRP & LTC & Ethereum Classic (ETC) \\
\hline $2017-04-01$ & BTC & ETH & XRP & LTC & DASH \\
\hline $2017-07-01$ & BTC & ETH & XRP & LTC & ETC \\
\hline $2017-09-01$ & BTC & ETH & XRP & LTC & DASH \\
\hline $2018-03-01$ & BTC & ETH & XRP & Bitcoin Cash (BCH) & LTC \\
\hline $2018-11-01$ & BTC & ETH & XRP & Stellar (XLM) & LTC \\
\hline $2019-01-01$ & BTC & ETH & XRP & BCH & EOS (EOS) \\
\hline
\end{tabular}

Table 1: Top 5 Eligible Cryptoassets - Monthly Reselection.

The crypto-basket composition is defined according to an Equally Weighted scheme, whose historical returns and volatility are studied towards the dynamic allocation between the cryptoassets and gold. We opt for a WRC allocation scheme between the two classes. Given the historical level of correlation between gold and crypto assets, an equal risk distribution among the two asset classes would be expected to be heavily concentrated towards gold as the lower volatility asset. Nevertheless, the chosen WRC setting, with a risk ratio that results to $80 \%$ of the total risk emanating from the crypto-basket component $(\alpha=4)$, ensures a good level of diversification, balancing the two components in the denominator of Eq. (3) as seen in Figure 3. 


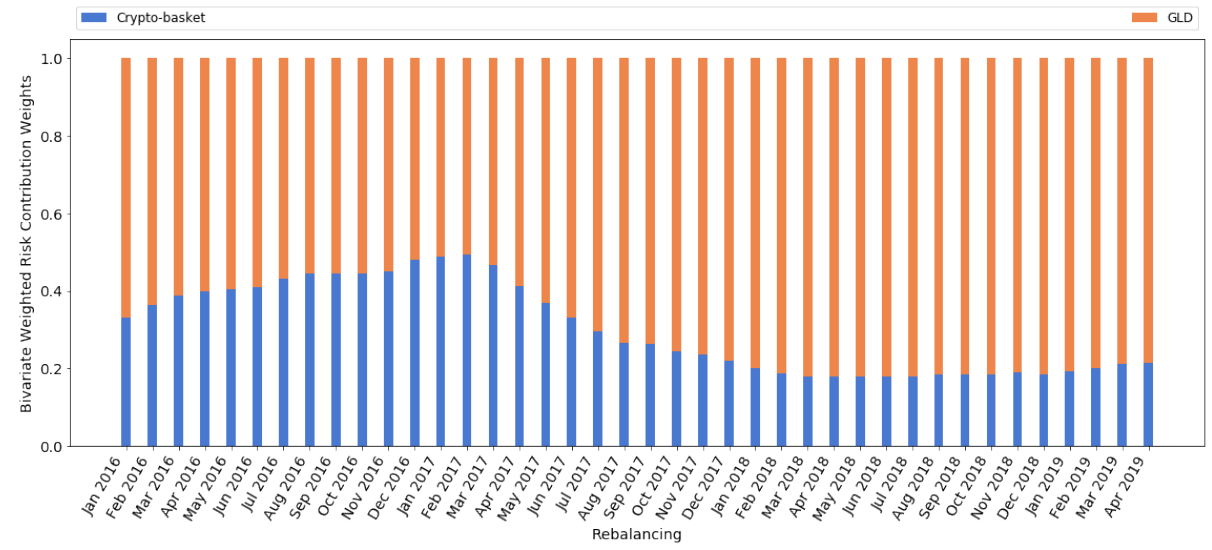

Fig. 3. Weighted Risk Contribution Allocation - EW Crypto Basket Base $-\alpha=4$

\subsection{Analysis and Results}

The results obtained from the bivariate WRC allocation with an EW cryptobasket base (WRC-EW Base) are directly compared with the following:

1. Bivariate WRC allocation with a 6-month rolling mean Market Capitalisation weighted crypto-basket base (WRC-MC Base)

2. Equally-weighted cryptoassets (EW)

3. Market capitalisation weighted cryptoassets (MC)

4. Equal Risk Contribution weighted cryptoassets (ERC)

5. Bitcoin and GLD weighted in accordance with the Shannon's Demon (SD)

6. Bitcoin only (BTC)

7. Gold only (GLD)

As seen in Table 2 and Figure 4, the proposed allocation scheme outperforms the rest in terms of historical risk-adjusted returns, as measured by the Sharpe Ratio. Moreover, a comparison with a typical index profile of the cryptoasset space, namely the MVIS Digital Assets 5 Index [14] (MVDA5) - a market capitalisation weighted index which tracks the performance of the five largest and most liquid cryptoassets - also reveals superiority in terms of the risk-return profile. Annualised returns are higher than a buy-and-hold GLD-only investment while annualised volatility levels are much lower than the crypto-market's. The ERC and EW present similar behaviour due to the assets' correlation structure; similarly, passive bitcoin and Market-Cap driven strategies do not reveal major differences. Table 2 also reports portfolio turnover, which reflects the total proportion of portfolio value traded (bought and sold) while rebalancing the portfolio, on an annualised basis as defined in [8].

Overall, the bivariate WRC allocation's performance is characterised by significantly lower volatility, and a more stable risk profile. The stability of the strategy's performance is further reflected in Figure 5. 


\begin{tabular}{||c|c|c|c|c||}
\hline Allocation Scheme & Annualised Returns & Annualised Volatility & Annualised Sharpe Ratio & Annualised Turnover \\
\hline \hline WRC-EW Base & 0.4797 & 0.2411 & 1.9894 & 1.6906 \\
\hline WRC-MC Base & 0.3199 & 0.2318 & 1.3800 & 1.1196 \\
\hline ERC & 0.9878 & 0.8062 & 1.2253 & 2.8512 \\
\hline EW & 1.0284 & 0.8292 & 1.2252 & 2.9556 \\
\hline MC & 0.6734 & 0.7560 & 0.8908 & 1.2240 \\
\hline SD & 0.4085 & 0.3889 & 1.0502 & 1.0800 \\
\hline BTC & 0.7629 & 0.7680 & 0.9934 & 0.0000 \\
\hline GLD & 0.0812 & 0.1452 & 0.5591 & 0.0000 \\
\hline MVDA5 & 1.1160 & 0.8757 & 1.2744 & Not Computed \\
\hline
\end{tabular}

Table 2: Annualised Performance of Allocation Schemes, Jan 2016-Apr 2019

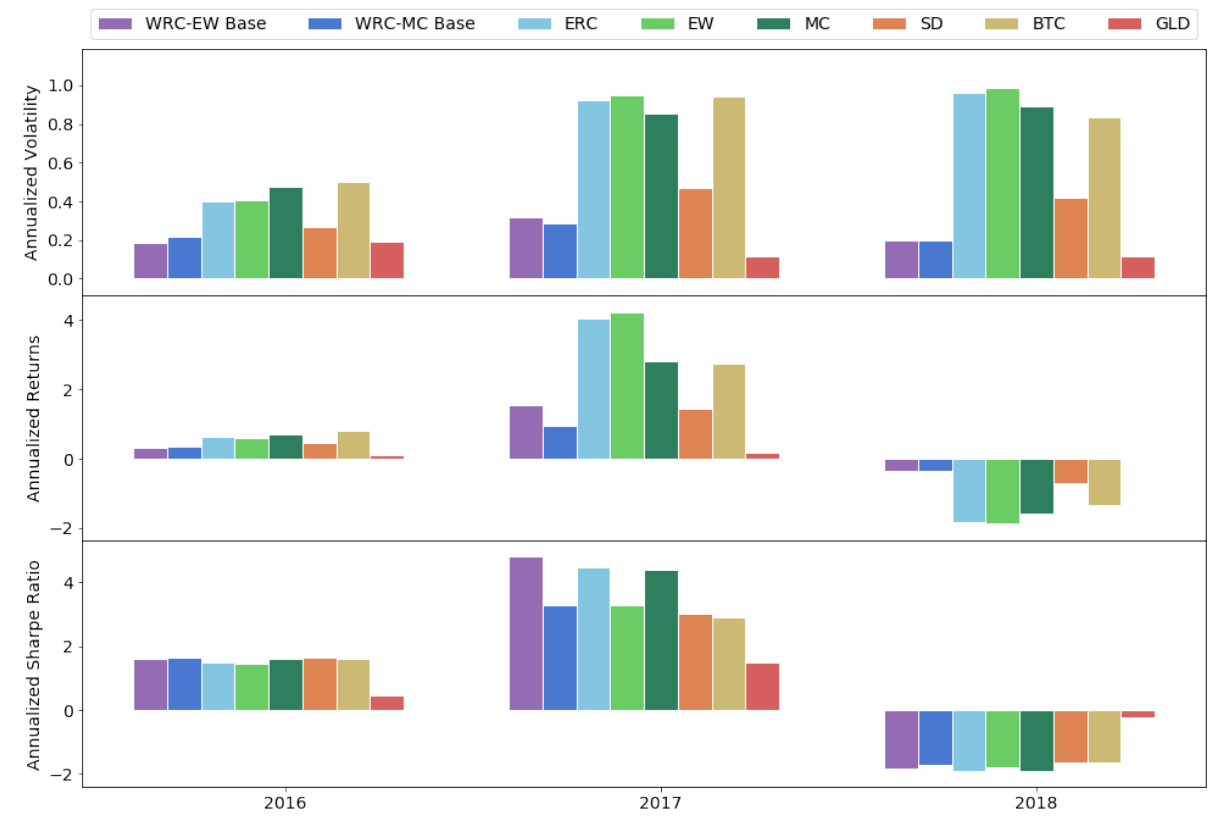

Fig. 4. Annualised Returns, Volatility \& Sharpe Ratio Jan 2016-Dec 2018 


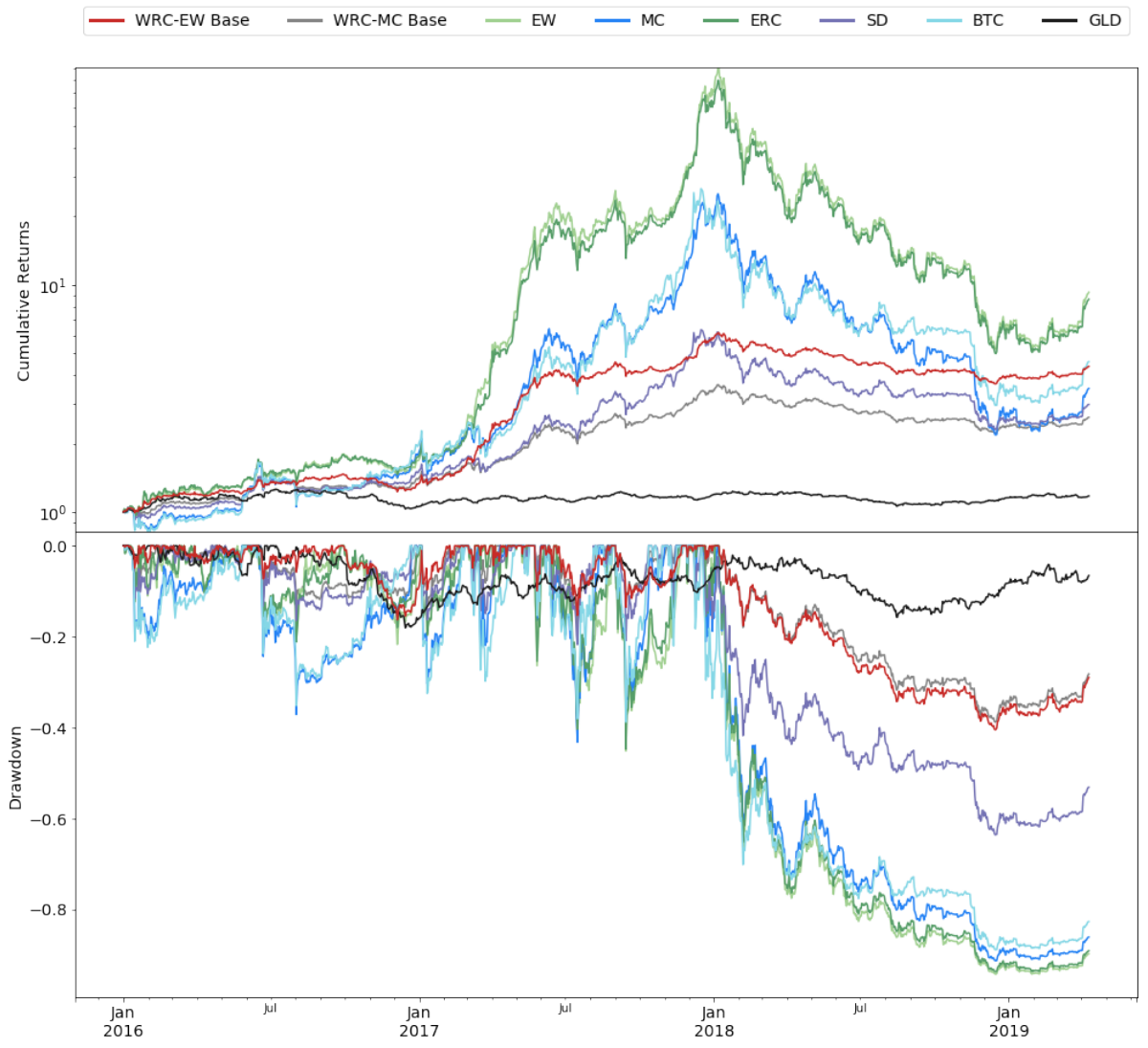

Fig. 5. Cumulative Returns \& Drawdown Jan 2016-Apr 2019

\section{Conclusion}

We have proposed the construction of an index that offers investors exposure to alternative assets. By exploiting the characteristics of the two asset classes of cryptoassets and gold - namely the extremely high volatility of the former, the low volatility of the latter and the lack of correlation between the two - it is characterised by an attractive ability to reduce price instability while raising the average return per unit of volatility. By generalising the theory of equal risk contribution, we offer a sophisticated, albeit intuitive, way of tuning the exposure of an index to uncorrelated asset classes. Another important feature of the index lies in the associated moderate turnover, which translates into moderate operating costs. Finally, by taking into account a variety of events unique to the cryptoasset space such as hard forks and airdrops and by proposing corresponding policies, we have designed an investable product whose distinctive elements make it a unique form of investment. 


\section{References}

1. Baur, D.G., Dimpfl, T., Kuck, K.: Bitcoin, gold and the US dollar - A replication and extension. Finance Research Letters 25, 103-110 (2018)

2. Bloomberg: Index methodology: Bloomberg Galaxy Crypto Index. Index Methodology (April 2019)

3. Booth, D., Fama, E.: Diversification returns and asset contributions. Financial Analysts Journal 48(3), 26-32 (1992)

4. Bouchey, P., Nemtchinov, V., Paulsen, A., Stein, D.: Volatility harvesting: Why does diversifying and rebalancing create portfolio growth? The Journal of Wealth Management 15(2), 26-35 (2012)

5. Bredin, D., Conlon, T., Poti, V.: Does gold glitter in the long-run? Gold as a hedge and safe haven across time and investment horizon. International Review of Financial Analysis 41, 320-328 (2015)

6. Burniske, C., Tatar, J.: Cryptoassets: The Innovative Investor's Guide to Bitcoin and Beyond. McGraw-Hill Education, New York, 1 edn. (2017)

7. CRYPTO20: CRYPTO20: The first tokenized cryptocurrency index fund. White Paper (October 2017)

8. Demiguel, V., Garlappi, L., Uppal, R.: Optimal versus naive diversification: How inefficient is the 1/n portfolio strategy? Review of Financial Studies 22 (05 2009)

9. Dubikovsky, V., Susinno, G.: Demystifying rebalancing premium and extending portfolio theory in the process (May 2015). https://doi.org/10.2139/ssrn.2927791

10. Dyhrberg, A.: Hedging capabilities of bitcoin. is it the virtual gold? Finance Research Letters 16(C), 139-144 (2016)

11. HM Treasury, Financial Conduct Authority, Bank of England: Cryptoassets task force: final report (October 2018)

12. Hood, M., Malik, F.: Is gold the best hedge and a safe haven under changing stock market volatility? Review of Financial Economics 22(2), 47-52 (2013)

13. Maillard, S., Roncalli, T., Teiletche, J.: On the properties of equally-weighted risk contributions portfolios (September 2008). https://doi.org/10.2139/ssrn.1271972

14. MV Index Solutions: MVIS CryptoCompare Digital Assets 5 Index. Index Factsheet (March 2019)

15. Poundstone, W.: Fortune's Formula. Hill and Wang (2005)

16. Rickards, J.: The New Case for Gold. Portfolio/Penguin Random House UK (2016)

17. Security and Exchange Commission: Virtual currencies: The oversight role of the U.S. Securities and Exchange Commission and the U.S. Commodity Futures Trading Commission. Report (February 2018)

18. Shu, J.J., Wang, Q.W.: Beyond Parrondo's Paradox. Scientific reports 4, 4244 (02 2014)

19. Stein, D., Nemtchinov, V., Pittman, S.: Diversifying and rebalancing emerging market countries. The Journal of Wealth Management 11(4), 79-88 (2009)

20. The Law Library of Congress: Regulation of cryptocurrency in selected jurisdictions. Report (June 2018)

21. Trimborn, S., Härdle, W.K.: CRIX an Index for Cryptocurrencies. Journal of Empirical Finance 49, 107-122 (December 2018)

\section{Acknowledgements}

Imperial College of London gratefully acknowledges the support given mutually by CoinShares and Elwood Capital Management. Their funding provides Imperial with the support needed to conduct the research found within this paper. 